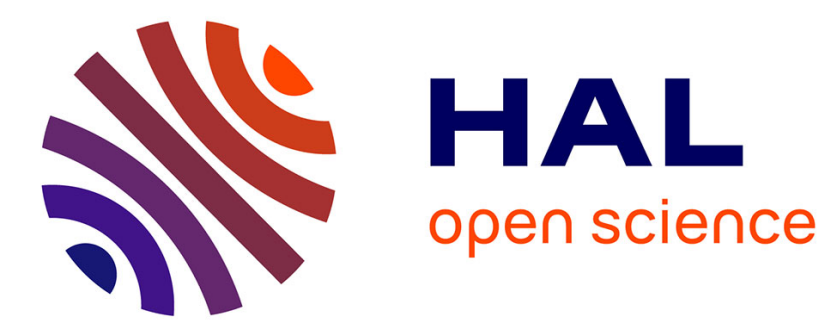

\title{
Custom on-axis head-mounted eye tracker for 3D active glasses
}

Vincent Nourrit, Rémi Poilane, Jean-Louis de Bougrenet de La Tocnaye

\section{To cite this version:}

Vincent Nourrit, Rémi Poilane, Jean-Louis de Bougrenet de La Tocnaye. Custom on-axis headmounted eye tracker for 3D active glasses. Electronic Imaging, Jan 2021, Online, United States. 10.2352/ISSN.2470-1173.2021.2.SDA-055 . hal-03208503

\section{HAL Id: hal-03208503 \\ https://hal.science/hal-03208503}

Submitted on 3 Sep 2021

HAL is a multi-disciplinary open access archive for the deposit and dissemination of scientific research documents, whether they are published or not. The documents may come from teaching and research institutions in France or abroad, or from public or private research centers.
L'archive ouverte pluridisciplinaire HAL, est destinée au dépôt et à la diffusion de documents scientifiques de niveau recherche, publiés ou non, émanant des établissements d'enseignement et de recherche français ou étrangers, des laboratoires publics ou privés. 


\title{
Custom on-axis head-mounted eye tracker for 3D active glasses
}

\author{
Vincent Nourrit; IMT Atlantique; Brest; France \\ Rémi Poilane; Eye Triple Shut; Brest; France \\ Jean-Louis de Bougrenet IMT Atlantique ; Brest ; France
}

\begin{abstract}
Currently, no low cost commercial $3 D$ active glasses with embedded eye tracker are available despite the importance of $3 D$ and eye tracking for numerous applications. In this context, a simple low cost eye tracker for $3 D$ glasses with liquid crystal shutters is presented and tested for orthoptics applications. By using a beam splitter to better align the camera with the line of sight when the subject looks at a target in front of him at far range, the new design allows recording high quality images with limited pupil deformation when compared to other commercial eye trackers where the cameras can be far from this axis (head mounted or fixed). Such a design could be useful for various applications from orthoptics to virtual reality
\end{abstract}

\section{Introduction}

Eye-tracking has a relatively long history dating back to the early 1800 s with direct observations of the eye. Already in 1901, Dodge [1] developed the first photograph that used corneal reflections to detect the gaze direction of the subject being examined. As time went by the technology improved to develop smaller and less intrusive eye tracking devices, culminating in today's wearable systems $[2,3]$.

A wide commercial offer exists nowadays reflecting the numerous possible applications of eye tracking. It ranges from high-end product such as the Tobii glasses 2 that can be used for a variety of studies, to trackers dedicated to a specific application (e.g. Gazelab, VisagraphTM) passing by more adaptable solutions (e.g. Pupil labs [4]). However, despite this wide range of products, no commercial 3D glasses with eye tracking are commercially available today. (By 3D glasses we refer here to active glasses for "classic" 3D display systems, and exclude virtual reality headset or augmented/mixt reality systems that are associated to different applications and for which embedded eye tracking systems can be found, e.g. FOVE in VR and Hololens v2 for AR). As 3D glasses become ubiquitous in numerous applications (entertainment, orthoptics, research in perception [5], etc.), the purpose of this study was to assess the benefits of a low-cost prototype eye tracker that works with conventional liquid crystal shutter glasses [6].

\section{Methods}

\subsection{Eye-tracker design}

A low-cost prototype eye tracker to be clipped on or embedded into $3 \mathrm{D}$ glasses has been developed for research purposes in 3D perception (cf. fig. 1). The 3D glasses used in this study rely on liquid crystal shutters and are manufactured by the company Eye Triple Shut (E3S) [6]. The eye tracker consists in an IR surveillance camera (ELP Full HD 1920x1080p), infrared LEDs (Vishay
VSMY12850 at $850 \pm 10 \mathrm{~nm}$ ) and a beam-splitter (BS) with a custom coating on each surface. The camera is mounted on the upper part of the glasses frame.

Eight (4 for each eye) LEDs are embedded into the eyewear frame and powered by a battery (also embedded into the frame). The LEDs are set to illuminate the eye uniformly. The LEDs irradiance in the cornea's plane is less than $2 \mathrm{~mW} / \mathrm{cm} 2$ which is well under ocular safety limits [7].

Contrary to most head mounted eye trackers, the camera does not point directly towards the eye but to the semi-reflecting plate (BS). This plate is positioned in front of the glasses using custom mechanical elements so as to direct the LEDs light reflected by the eye towards the camera as if the camera was placed in front of the eyes, on the line of sight (Fig. 1). The line of sight of the eye is defined here as the line passing by the center of the pupil to the object of regard when this object is placed at far distance in front of the subject. The plate holder tilt and axial position in front of the frame is adjustable to allow for an easy adjustment of the camera with the eye. The custom coating is made to maximize reflections at $850 \mathrm{~nm}$ and minimize reflections in the visible part of the spectrum to avoid visual artefacts (Fig. 2). Figure 3 depicts a monocular configuration but extension to a binocular one is straightforward. In this configuration, the BS size $(6 \times 3.5 \mathrm{~cm})$ limits the field of view to approximately $\left(63 \times 50^{\circ}\right)$. Compared to more compact commercial alternatives, the eye tracker is slightly heavier $(70 \mathrm{~g}$ when SMI and Tobii glasses weight approximately $45 \mathrm{~g}$ ) and costs a fraction of the price (approximately $1 \mathrm{k}$ versus $15 \mathrm{k}$ for the SMI or Tobii glasses) of which the coating accounts for approximately $90 \%$ (which could be easily reduced by mass production).

The choice of using a BS rather than pointing the camera directly towards the eye was motivated by several factors. Firstly finding a low cost, fast $(>120 \mathrm{~Hz})$, light sensitive camera (to respect ocular safety limits), small enough to fit on the glasses, and with good quality optics is a difficult task. Placing the camera as in Fig. 1 reduces the size constraint which significantly increases the choice and number of possible cameras and allow to choose cameras with a low f-number optics. Secondly, a close distance and a strong tilt between the eye (i.e. the object) and the camera are obviously not ideal imaging conditions. Placing the camera "on axis", i.e. in front of the eye, on the line of sight, at a larger distance allows imaging the pupil with less distortion than placed for instance right under the eye. This is important as pupil shape can influence the parameter extraction and hence the eye tracker's performances $[8,9]$. Thirdly, our design allows using only one camera for both eye to avoid synchronization issues (at the expense of a lower resolution per eye). The single camera could image directly both eyes (thus losing the alignment) or the alignment could be preserved by using a periscopic optics for each eye [10]. 


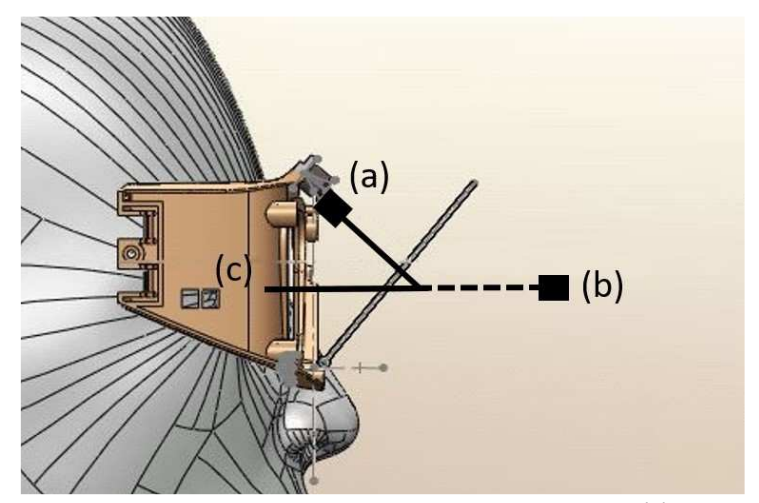

Figure 1: The beam splitter allows to better align the camera (a) with the line of sight (b). (c) denotes the virtual position of the camera

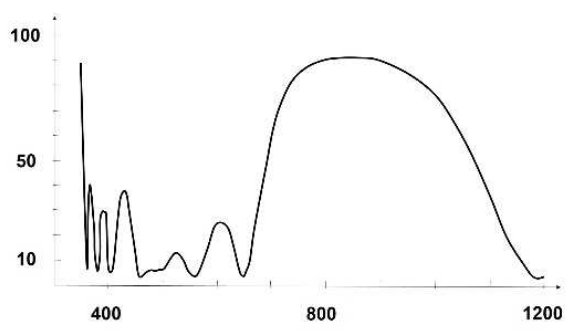

Figure 2: Spectral reflectance (\%) of the beam splitter's coating (camera side) as a function of the wavelength $(\mathrm{nm})$. Units for abscissa and ordinate axis are respectively $\mathrm{nm}$ and \%.

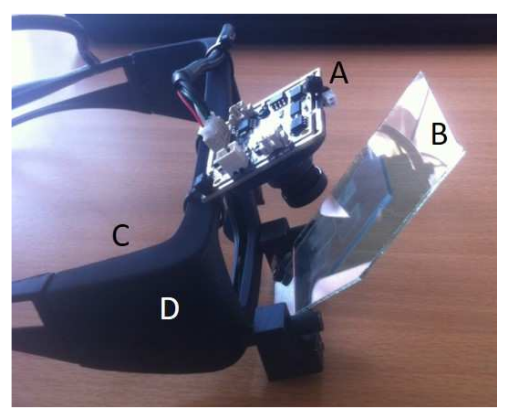

Figure 3: The prototype eye tracker where $A$ ) is the camera, $B$ ) the beam splitter $(B S), C)$ the $3 D$ glasses and $D$ ) where the battery and most of the LC shutters' electronics are embedded. In this picture, the LC shutters are absent

\subsection{Comparison method}

A usual difficulty when comparing eye tracker is to correlate their respective measurements [11]. In addition, in this study, no dedicated software has been developed for our prototype. For this reason and because its main advantage is to allow for better imaging conditions by folding the optical path, we chose to compare the image quality and pupil deformation obtained with this prototype to those obtained with 3 commercial eye trackers: two head mounted ones (Tobii Pro Glasses 2, SMI Glasses) and the desktop based Facelab5. Characteristics of the 4 eye trackers are reported in Table
1. Images of the eye were registered for different gaze angles $\left(30^{\circ}\right.$ temporal, $30^{\circ}$ downwards, $30^{\circ}$ upwards), different lighting conditions (140lux, 600lux), and different LC shutters configuration (no shutters, with closed shutters). The three different gaze angles correspond to large values where eye trackers capacities can be challenged. The low lighting condition correspond to the one of an optometry box (the first application we considered was for orthoptics) and the high one (600 lux) where obtained indoor but with bright lights on and sitting by the window. As the other eye trackers do not have LC shutters, we placed one shutter in front of the eyewear to operate in a comparable condition of use (tacking care of not affecting the led illumination). We did not report here images recorded with opened shutters as it would provide limited information when compared to the two other cases.

\begin{tabular}{|l|c|c|c|c|}
\hline & $\begin{array}{c}\text { Freq. } \\
(\mathbf{H z})\end{array}$ & $\begin{array}{c}\text { Angular } \\
\text { range }\end{array}$ & $\begin{array}{c}\text { Irradiance } \\
(\mu \mathrm{W} / \mathbf{m m 2})\end{array}$ & $\begin{array}{c}\text { Wavelength } \\
(\mathbf{n m})\end{array}$ \\
\hline $\begin{array}{l}\text { Tobii } \\
\text { glasses 2 }\end{array}$ & 100 & $160^{\circ} \times 70^{\circ}$ & $\geq 6$ & 860 \\
\hline $\begin{array}{l}\text { SMI } \\
\text { glasses }\end{array}$ & 120 & $80^{\circ} \times 60^{\circ}$ & 1 & 985 \\
\hline Facelab 5 & 60 & $45^{\circ} \times 22^{\circ}$ & 1 & $850 / 875$ \\
\hline Prototype & 120 & $\begin{array}{c}63^{\circ} \times 50^{\circ} \\
\mathrm{monocular}\end{array}$ & 2 & 850 \\
\hline
\end{tabular}
commercial eye trackers, the frequency and the angular range in which the eye tracker works come from the manufacturer's specifications. The wavelength used to illuminate the eye and the associated corneal irradiance were measured.

\section{Results}

In practice, raw images from commercial eye trackers are not directly accessible and the only ones we could use are the ones presented in the user interface associated with each eye tracker. Therefore, no information is available on the resolution of the eye tracking camera and the processing that may have been applied to these images. Consequently, image quality can only be assessed subjectively assuming that the best possible images are displayed in the user interface. A Matlab script was used to calculate the ellipticity , or "flattening" $(\varepsilon)$ of the pupil in each image, defined as $\varepsilon=1-b / a$ where $a$ and $b$ are respectively the semi-major and semiminor axis. Images obtained for the different gaze directions are presented respectively in Fig. 4. Results for pupil deformation are presented in table 2 .

\begin{tabular}{|l|l|l|l|l|l|l|}
\hline & \multicolumn{2}{|l|}{$30^{\circ}$ temp } & \multicolumn{2}{l|}{$30^{\circ}$ upwards } & \multicolumn{2}{l|}{$30^{\circ}$ downwards } \\
\hline $\begin{array}{l}\text { Lighting } \\
\text { Conditions }\end{array}$ & $\begin{array}{l}140 \\
\text { lux }\end{array}$ & $\begin{array}{l}600 \\
\text { lux }\end{array}$ & $\begin{array}{l}140 \\
\text { lux }\end{array}$ & $\begin{array}{l}600 \\
\text { lux }\end{array}$ & $\begin{array}{l}140 \\
\text { lux }\end{array}$ & $\begin{array}{l}600 \\
\text { lux }\end{array}$ \\
\hline Facelab & 0.35 & 0.3 & 0.18 & 0.08 & 0.04 & 0.11 \\
\hline Prototype & 0 & 0 & 0.17 & 0.18 & 0.13 & 0.11 \\
\hline Tobii & 0.34 & 0.33 & 0.57 & 0.52 & 0.04 & 0 \\
\hline SMI & 0.33 & 0.38 & 0.52 & 0.64 & 0.03 & 0.02 \\
\hline
\end{tabular}

Table 2: Pupil ellipticity (calculated for images obtained without shutters)

Regarding the influence of the LC shutters: the Facelab uses infrared light for which the shutters are mostly transparent $(>70 \%)$. The shutter's influence was then thus limited to reducing the amount of light illuminating the eye (as illustrated by the pupil dilatation in Fig. 
4) and possibly slightly degrading the image quality (increased scattering). As a result, the pupil may be more difficult to detect (Fig. 4), leading to calibration difficulties and incorrect tracking. For the head mounted trackers, light reflections on the shutters had not significant effect.

With respect to lighting conditions, it had a limited impact on the image quality as all eye trackers probably use a band pass filter to limit the influence of external light sources and/or automatically enhance the images' contrast.

With regards to pupil shape, pupil ellipticity values (table 2) are in agreement with expectations. When using the prototype, a careful adjustment of the mirror is necessary to avoid that the eyelid intersects the pupil, particularly for downwards direction of gaze. A problem that the Tobii or SMI glasses avoid with cameras placed under the eyes. As a result, the pupil appears the most distorted when imaged with the SMI and Tobii for the upwards direction of gaze. Once this adjustment done, our prototype provides excellent images (pupil entirely imaged and with limited deformation, i.e. low ellipticity values) in all directions of gaze when compared to the commercial eye trackers.

In addition to assessing image quality and pupil deformation, we also verified that the prototype's illumination system did not disrupt the normal functioning of the glasses (since E3S' glasses, as many 3D shutter glasses, uses IR-based communication for the shutter synchronization). We thus used the eye tracker during a standard orthoptics convergence exercise where the target was projected with a 3D projector (Orthoptica ${ }^{\circledR}$ ). Because the E3S' synchronization uses a modulated signal in another spectral band $(>900 \mathrm{~nm})$ than the one used for illumination, this last one did not have any impact on image stability, image drift, or on/off shutter commuting time. The feedback from the orthoptist in charge of the test regarding the possibility to observe and record on his computer the subject's eye during the exercise was very positive.
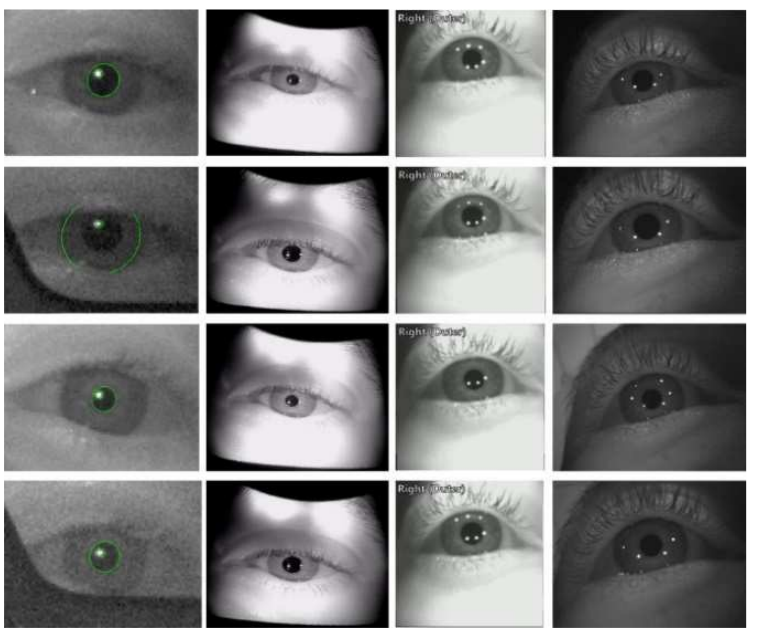
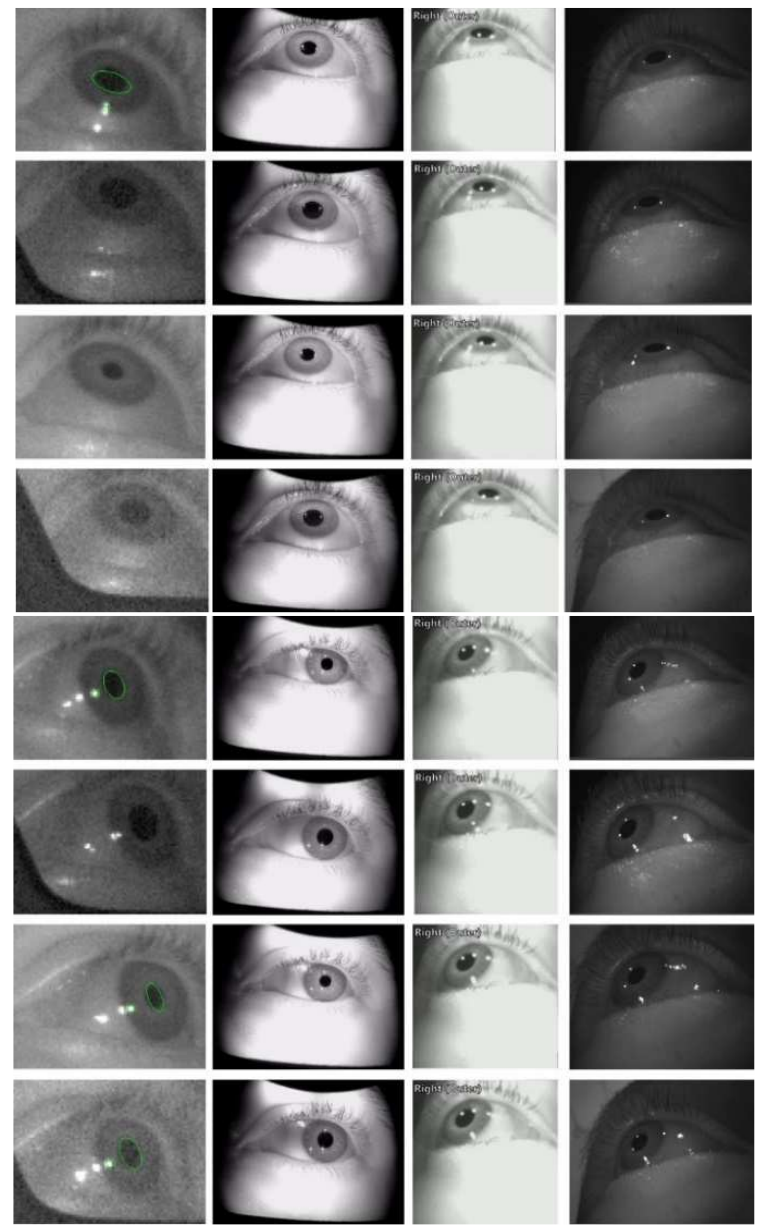

Figure 4: Images of the left eye obtained with the different eye trackers when looking at a target situated $30^{\circ}$ downwards (1st 4 rows), upwards (rows 5-8), temporal (last 4 rows). Images from left to right were respectively recorded with the Facelab, prototype, Tobii, SMI eye trackers. Imaging conditions from first row to bottom row were respectively: no shutters/140lux, shutters closed/140lux, no shutters/600lux, shutters closed/600lux.

\section{Discussion}

We have presented and assessed an eye tracker for LC 3D glasses. The quality of the image was the main focus of the study and we demonstrated that this low cost prototype provides high quality images when compared to commercial products (reduced pupil deformation, no impact of shutters). In this prototype, the key element is a beam splitter used to better align the camera with the line of sight (as defined in section 2), thus allowing for better imaging conditions as illustrated by the minimum pupil deformation for large gaze angles. This in turn could facilitate pupil segmentation with a positive impact on the required processing power and improved tracking performances. Such design could be useful for applications such as orthoptics where orthoptists require seeing the patient's eye and have limited requirements in terms of compactness when compared to other fields (marketing, sport). The arrangement 
could be easily designed as a removable clip-on optics or also adapted for used in VR helmets.

\section{References}

[1] R. Dodge, TS. Cline. The angle velocity of eye movements. Psychological Review 8(2), 145-157, 1901.

[2] JL de Bougrenet de la Tocnaye, L. Dupont, F. Ferranti, C. Lahuec, V. Nourrit, F. Seguin and L. Adam. A wireless contact lens eye tracking system WIRCLEY project (example of a smart sensors development platform). 5th International Conference SENSO Workshop November 15th to 17th, 2017, Gardanne (France)

[3] C. Agell, P. Casale, G. Squillace. Method and a system for monitoring an eye position. US Patent 20,180,341,328 A1, 2017.

[4] M. Kassner, W. Patera, Andreas Bulling. Pupil: an open source platform for pervasive eye tracking and mobile gaze-based interaction. Published in UbiComp Adjunct. DOI:10.1145/2638728.2641695, 2014.

[5] D. Zhang, V. Nourrit, JL de Bougrenet de la Tocnaye. Enhancing motion-in-depth perception of random-dot stereograms, Perception 47(7):722-734, 2018.
[6] A. Srivastava, JL de Bougrenet de la Tocnaye, L. Dupont. Liquid crystal active glasses for 3D cinema, Journal of display technology 6(10):522-30, 2010.

[7] FC Delori, RH Webb, D. Sliney. Maximum permissible exposures for ocular safety (ANSI 2000), with emphasis on ophthalmic devices. JOSA A 24(5) : 1250-65, 2007.

[8] M. Nyström, I. Hooge, R. Andersson. Pupil size influences the eyetracker signal during saccades. Vision Res. 121(4):95-103, 2016.

[9] KW Choe, R. Blake, SH Lee. Pupil size dynamics during fixation impact the accuracy and precision of video-based gaze estimation. Vision Res. 118(1):48-59, 2016.

[10] JL de Bougret de la Tocnaye, N. Fraval, D. Stoenescu, K. Sathaye, F. Lucarz. Stereoscopic image capturing device i.e. three dimensional web camera, for use on e.g. cell phone, for producing e.g. film for cinema or TV, has adaptive optical elements whose focal distance and line of sight orientation can be adjusted. FR2970573 (A1), 2012.

[11] J. Titz, A. Scholz, P. Sedlmeier. Comparing eye trackers by correlating their eye-metric data. Behav Res Methods. 50(5):1853$1863,2018$. 